\title{
The Improved Generalized tanh-coth Method Applied to Sixth-Order Solitary Wave Equation
}

\author{
M. Torvattanabun, J. Simmapim, D. Saennuad, and T. Somaumchan \\ Department of Mathematics, Faculty of Science and Technology, Loei Rajabhat University, Loei 42000, Thailand \\ Correspondence should be addressed to M. Torvattanabun; montri_kai@windowslive.com
}

Received 10 February 2017; Revised 1 May 2017; Accepted 29 May 2017; Published 17 July 2017

Academic Editor: Emir Köksal

Copyright ( 2017 M. Torvattanabun et al. This is an open access article distributed under the Creative Commons Attribution License, which permits unrestricted use, distribution, and reproduction in any medium, provided the original work is properly cited.

The improved generalized tanh-coth method is used in nonlinear sixth-order solitary wave equation. This method is a powerful and advantageous mathematical tool for establishing abundant new traveling wave solutions of nonlinear partial differential equations. The new exact solutions consisted of trigonometric functions solutions, hyperbolic functions solutions, exponential functions solutions, and rational functions solutions. The numerical results were obtained with the aid of Maple.

\section{Introduction}

Nonlinear evolution equations (NLEEs) play an important role in various branches of scientific disciplines, such as fluid mechanics, optical fibers, plasma physics, chemical physics, biology, solid state physics, oceans engineering, and many other scientific applications. The solitary wave was introduced by Russell more than a century ago [1]. In the past years, many powerful methods for finding exact solutions of NLEEs have been proposed, such as the generalized $\left(G^{\prime} / G\right)$-expansion method [2], the tanh-coth method [3], the modified sine-cosine method [4], the generalized unified method [5], the improved F-expansion method [6], the generalized Kudryashov method [7], the generalized Riccati equation mapping method [8], the modified Kudryashov method [9], the $\exp (-\phi(\xi))$ method [10], the lie symmetry analysis method [11], the first integral method [12], and the consistent Riccati expansion [13].

Another powerful method has been presented by Malfliet [14], who had customized the tanh technique and called the tanh method. In 2002, Fan and Hona [15] extended the tanh method which is called the extended tanh method, by using $U(\xi)=\sum_{k=0}^{M} a_{k} Y^{k}$ as traveling wave solutions. In 2007, Wazwaz [3] extended and improved this method which is called the tanh-coth method. In this method $U(\xi)=$ $\sum_{k=0}^{M} a_{k} Y^{k}+\sum_{k=1}^{M} b_{k} Y^{-k}$ is used as traveling wave solutions. In 2008 Gómez and Salas [16] improved and generalized this method which is called the improved generalized tanh-coth method, by using $U(\xi)=\sum_{i=0}^{M} a_{i} \phi(\xi)^{i}+\sum_{i=M+1}^{2 M} a_{i} \phi(\xi)^{M-i}$, where $\phi$ is the solution of the generalized Riccati equation. Afterwards, several researchers applied this method to obtain new exact solutions for nonlinear PDEs [17-20].

In 2017, Christou [21] studies solitons occurring in electrical nonlinear transmission lines; there are called electrical solitons. The problem is applied to Ohm's law of solid state physics by using Taylor-series expansions.

In this paper, we focus on using the improved generalized tanh-coth method for finding exact solutions of the sixthorder solitary wave equation:

$$
V_{t t}=\left[a V+b V^{2}-c V^{3}+g V_{x x}+h V x x x x\right]_{x x},
$$

which was proposed by Christou [21] and

$$
\begin{aligned}
& a=\frac{h^{2}}{C_{0} L} \\
& b=\frac{1}{2 F_{0}} \\
& c=\frac{1}{3 F_{0}^{2}}
\end{aligned}
$$




$$
\begin{aligned}
& g=\frac{(12 \delta+1) h^{4}}{12 C_{0} L} \\
& h=\frac{(60 \delta+1) h^{6}}{360 C_{0} L} .
\end{aligned}
$$

In Section 2, we briefly describe the improved generalized tanh-coth method; in Section 3, the improved generalized tanh-coth method is applied to the sixth-order solitary wave equations. The last section is short summary and discussion.

\section{The Improved Generalized tanh-coth Method}

Consider the nonlinear partial differential equation in the variables $x$ and $t$

$$
P_{1}\left(u, u_{x}, u_{t}, u_{x, t}, u_{x, x}, u_{t, t}, \ldots\right)=0
$$

The traveling wave transformation is given by

$$
u(x, t)=U(\xi), \quad \xi=x-\lambda t+\xi_{0}
$$

where $\lambda$ is the wave speed. We can reduce (3) to the ordinary differential equation

$$
P_{2}\left(U, U^{\prime}, U^{\prime \prime}, \ldots\right)=0
$$

According to the improved generalized tanh-coth method, we seek the exact solution of (3) that can be expressed in the following form:

$$
U(\xi)=\sum_{i=0}^{M} a_{i} \phi(\xi)^{i}+\sum_{i=M+1}^{2 M} a_{i} \phi(\xi)^{M-i}
$$

where $M$ is a positive integer that will be determined by balancing the highest order derivative term with the highest order nonlinear term. The coefficients $a_{i}$ are constants $\left(a_{M} \neq\right.$ 0 and $a_{-M} \neq 0$ ) that are determined later while the new variable $\phi(\xi)$ is the solution to the generalized Riccati equation

$$
\phi^{\prime}(\xi)=\alpha+\beta \phi(\xi)+\gamma(\phi(\xi))^{2},
$$

where $\alpha, \beta$, and $\gamma$ are constants. The solutions of generalized Riccati equation are given by [18].

Case 1 (exponential function solutions). When $\alpha=0$

$$
\phi(\xi)=\frac{\beta}{-\gamma+\beta e^{-\beta \xi}} .
$$

Case 2 (trigonometric and hyperbolic function solution). When $\beta=0$,

$$
\phi(\xi)= \begin{cases}\frac{\sqrt{\alpha \gamma}}{\gamma} \tan (\sqrt{\alpha \gamma} \xi), & \alpha>0, \gamma>0, \\ \frac{\sqrt{\alpha \gamma}}{\gamma} \tanh (\sqrt{\alpha \gamma} \xi), & \alpha>0, \gamma<0, \\ \frac{\sqrt{-\alpha \gamma}}{\gamma} \tanh (-\sqrt{-\alpha \gamma} \xi), & \alpha<0, \gamma>0, \\ \frac{\sqrt{\alpha \gamma}}{\gamma} \tan (-\sqrt{\alpha \gamma} \xi), & \alpha<0, \gamma<0 .\end{cases}
$$

Case 3 (exponential function solutions). When $\gamma=0$,

$$
\phi(\xi)=\frac{-\alpha+\beta e^{\beta \xi}}{\beta} .
$$

Case 4 (rational function solution). When $\alpha=\beta=0$,

$$
\phi(\xi)=-\frac{1}{\gamma \xi} .
$$

Case 5 (rational function solution). When $\beta^{2} \neq 0$ and $\beta^{2}=$ $4 \alpha \gamma$,

$$
\phi(\xi)=-\frac{2 \alpha(\beta \xi+2)}{\beta^{2} \xi} .
$$

Case 6 (trigonometric function solution). When $\beta^{2}<4 \alpha \gamma$ and $\gamma \neq 0$,

$$
\phi(\xi)=\frac{\sqrt{4 \alpha \gamma-\beta^{2}} \tan \left((1 / 2) \sqrt{4 \alpha \gamma-\beta^{2}} \xi\right)-\beta}{2 \gamma} .
$$

Case 7 (hyperbolic function solution). When $\beta^{2}>4 \alpha \gamma$ and $\gamma \neq 0$,

$$
\phi(\xi)=\frac{\sqrt{\beta^{2}-4 \alpha \gamma} \tanh \left((1 / 2) \sqrt{\beta^{2}-4 \alpha \gamma \xi}\right)-\beta}{2 \gamma} .
$$

We substitute (6) into (5) and collect all terms with the same order of $\phi^{j}(\xi)$; we get a polynomial in $\phi(\xi)$. Equating each coefficient of the polynomial to zero, we will give a system of algebraic equations involving the parameters $a_{i}, \alpha$, $\gamma$, and $\beta$. Solving the system, we can construct a variety of exact solutions of (5).

\section{The Improved Generalized tanh-coth Method Applied to Sixth-Order Solitary Wave Equation}

We use the wave transformations $V(x, t)=V(\xi), \xi=x-\lambda t+$ $\xi_{0}$, to reduce (1) to the following ODE:

$$
\begin{gathered}
\left(\lambda^{2}-a\right) V^{\prime \prime}(\xi)-b\left(V^{2}(\xi)\right)^{\prime \prime}+c\left(V^{3}(\xi)\right)^{\prime \prime} \\
+g V^{\prime \prime \prime \prime}(\xi)-h V^{\prime \prime \prime \prime \prime \prime \prime}(\xi)=0 .
\end{gathered}
$$


Balancing the highest order term $V^{\prime \prime \prime \prime \prime \prime \prime ~}$ with the highest order nonlinear term $\left(V^{3}\right)^{\prime \prime \prime}$ in (13), we have

$$
M+6=3 M+2
$$

then $M=2$. Consequently, we set

$$
\begin{aligned}
V(\xi)= & a_{0}+a_{1} \phi(\xi)+a_{2}(\phi(\xi))^{2}+a_{3}(\phi(\xi))^{-1} \\
& +a_{4}(\phi(\xi))^{-2}
\end{aligned}
$$

Using (6) and (14) in (13) and equating all the coefficients of power of $\phi(\xi)$ to be zero, we obtain a system of algebraic equations in the unknowns $a_{0}, a_{1}, a_{2}, a_{3}, a_{4}, \lambda, \alpha, \beta$, and $\gamma$.

$$
\begin{aligned}
& \phi(\xi)^{-8}:-5040 \alpha^{6} z a_{4}+42 \alpha^{2} c a_{4}^{3}=0, \\
& \phi(\xi)^{-7}:-720 \alpha^{6} z a_{3}-19440 \alpha^{5} \beta z a_{4}+90 \alpha^{2} c a_{3} a_{4}^{2} \\
& +8 \alpha \beta c a_{4}^{3}=0, \\
& \phi(\xi)^{-6}: 165 c a_{3} a_{4}^{2} \alpha \beta+72 c a_{4}^{3} \alpha \gamma+60 c a_{0} a_{4}^{2} \alpha^{2} \\
& +60 c a_{3}^{2} \alpha^{2} a_{4}-29400 z a_{4} \alpha^{4} \beta^{2}-13440 z a_{4} \alpha^{5} \gamma \\
& -2520 z a_{3} \alpha^{5} \beta+36 c a_{4}^{3} \beta^{2}-20 b a_{4}^{2} \alpha^{2} \\
& -120 g a_{4} \alpha^{4}=0 \text {, } \\
& \phi(\xi)^{-5}: 75 c a_{3} a_{4}^{2} \beta^{2}+66 c a_{4}^{3} \beta \gamma+72 c a_{0} a_{3} a_{4} \alpha^{2} \\
& +108 c a_{0} a_{4}^{2} \alpha \beta+150 c a_{3} a_{4}^{2} \alpha \gamma+108 c a_{3}^{2} a_{4} \alpha \beta \\
& -38640 z a_{4} \alpha^{4} \beta \gamma-24 b a_{3} a_{4} \alpha^{2}-36 b a_{4}^{2} \alpha \beta \\
& +36 c a_{1} a_{4}^{2} \alpha^{2}-21840 z a_{4} \alpha^{3} \beta^{3}-3360 z a_{3} \alpha^{4} \beta^{2} \\
& -1680 z a_{3} \alpha^{5} \gamma-336 g a_{4} \alpha^{3} \beta+12 c a_{3}^{3} \alpha^{2} \\
& -24 g a_{3} \alpha^{4}=0 \text {, } \\
& \phi(\xi)^{-4}: 96 c a_{0} a_{4}^{2} \alpha \gamma+36 c a_{1} a_{3} a_{4} \alpha^{2}+135 c a_{3} a_{4}^{2} \beta \gamma \\
& -4200 z a_{3} \alpha^{4} \beta \gamma-40152 z a_{4} \alpha^{3} \beta^{2} \gamma-42 b a_{3} a_{4} \alpha \beta \\
& +96 c a_{3}^{2} a_{4} \alpha \gamma+63 c a_{1} a_{4}^{2} \alpha \beta-12096 z a_{4} \alpha^{4} \gamma^{2} \\
& -8106 z a_{4} \alpha^{2} \beta^{4}-2100 z a_{3} \alpha^{3} \beta^{3}-240 g a_{4} \alpha^{3} \gamma \\
& -330 g a_{4} \alpha^{2} \beta^{2}-60 g a_{3} \alpha^{3} \beta+21 c a_{3}^{3} \alpha \beta+48 c a_{3}^{2} a_{4} \\
& -12 b a_{0} a_{4} \alpha^{2}-32 b a_{4}^{2} \alpha \gamma+18 c a_{0}^{2} a_{4} \alpha^{2}+18 c a_{0} a_{3}^{2} \alpha^{2} \\
& +48 c a_{0} a_{4}^{2} \beta^{2}+18 c a_{2} a_{4}^{2} \alpha^{2}-6 a a_{4} \alpha^{2}+6 \lambda^{2} a_{4} \alpha^{2} \\
& +30 c a_{4}^{3} \gamma^{2}-6 b a_{3}^{2} \alpha^{2}-16 b a_{4}^{2} \beta^{2} \\
& +126 c a_{0} a_{3} a_{4} \alpha \beta=0 \text {, }
\end{aligned}
$$

$\phi(\xi)^{-3}: 54 c a_{0} a_{3} a_{4} \beta^{2}+84 c a_{0} a_{4}^{2} \beta \gamma+12 c a_{2} a_{3} a_{4} \alpha^{2}$

$+30 c a_{2} a_{4}^{2} \alpha \beta-20 b a_{0} a_{4} \alpha \beta-440 g a_{4} \alpha^{2} \beta \gamma$

$-3584 z a_{3} \alpha^{3} \beta^{2} \gamma-17920 z a_{4} \alpha^{2} \beta^{3} \gamma$

$-22960 z a_{4} \alpha^{3} \beta \gamma^{2}-36 b a_{3} a_{4} \alpha \gamma+30 c a_{0}^{2} a_{4} \alpha \beta$

$+54 c a_{1} a_{4}^{2} \alpha \gamma+84 c a_{3}^{2} a_{4} \beta \gamma+12 c a_{0} a_{1} a_{4} \alpha^{2}$

$+30 c a_{0} a_{3}^{2} \alpha \beta-1330 z a_{4} \beta^{5} \alpha-1232 z a_{3} \alpha^{4} \gamma^{2}$

$-602 z a_{3} \alpha^{2} \beta^{4}-130 g a_{4} \alpha \beta^{3}-40 g a_{3} \alpha^{3} \gamma$

$-50 g a_{3} \alpha^{2} \beta^{2}+18 c a_{3}^{3} \alpha \gamma+60 c a_{3} a_{4}^{2} \gamma^{2}+10 a_{4} \lambda^{2} \alpha \beta$

$-4 b a_{0} a_{3} \alpha^{2}-10 a a_{4} \alpha \beta-4 b a_{1} a_{4} \alpha^{2}-10 b a_{3}^{2} \alpha \beta$

$-18 b a_{3} a_{4} \beta^{2}-28 b a_{4}^{2} \beta \gamma+6 c a_{0}^{2} a_{3} \alpha^{2}+2 c a_{1} a_{4}^{2} \beta^{2}$

$+6 c a_{1} a_{3}^{2} \alpha^{2}-2 a a_{3} \alpha^{2}+2 a_{3} \lambda^{2} \alpha^{2}+9 c a_{3}^{3} \beta^{2}$

$+108 c a_{0} a_{3} a_{4} \alpha \gamma+60 c a_{1} a_{3} a_{4} \alpha \beta=0$,

$\phi(\xi)^{-2}: 24 c a_{0} a_{3}^{2} \alpha \gamma+9 c a_{1} a_{3}^{2} \alpha \beta+24 c a_{1} a_{3} a_{4} \beta^{2}$

$+24 c a_{2} a_{4}^{2} \alpha \gamma-6 b a_{0} a_{3} \alpha \beta-16 b a_{0} a_{4} \alpha \gamma$

$-232 g a_{4} \alpha \gamma \beta^{2}-60 g a_{3} \alpha^{2} \beta \gamma-116 z a_{3} \alpha^{2} \beta \gamma$

$-1848 z a_{3} \alpha^{3} \beta \gamma^{2}-13320 z a_{4} \alpha^{2} \beta^{2} \gamma^{2}$

$-3096 z a_{4} \alpha \beta^{2} \gamma-30 b a_{3} a_{4} \beta \gamma-6 b a_{1} a_{4} \alpha \beta$

$+9 c a_{0}^{2} a_{3} \alpha \beta+24 c a_{0}^{2} a_{4} \alpha \gamma+45 c a_{1} a_{4}^{2} \beta \gamma$

$-3968 z a_{4} \alpha^{3} \gamma^{3}-63 z a_{3} \beta^{5} \alpha-136 g a_{4} \alpha^{2} \gamma^{2}$

$-15 g a_{3} \alpha \beta^{3}+15 c a_{3}^{3} \beta \gamma+36 c a_{3}^{2} a_{4} \gamma^{2}+3 a_{3} \lambda^{2} \alpha \beta$

$+8 a_{4} \lambda^{2} \alpha \gamma-8 b a_{0} a_{4} \beta^{2}-3 a a_{3} \alpha \beta-8 a a_{4} \alpha \gamma$

$-8 b a_{3}^{2} \alpha \gamma+12 c a_{0}^{2} a_{4} \beta^{2}+12 c a_{0} a_{3}^{2} \beta^{2}+36 c a_{0} a_{4}^{2} \gamma^{2}$

$+12 c a_{2} a_{4}^{2} \beta^{2}-4 a a_{4} \beta^{2}+4 a_{4} \lambda^{2} \beta^{2}-4 a_{3}^{2} b \beta^{2}$

$-12 b a_{4}^{2} \gamma^{2}-64 z a_{4} \beta^{6}-16 g a_{4} \beta^{4}+90 c a_{0} a_{3} a_{4} \beta \gamma$

$+18 c a_{0} a_{1} a_{4} \alpha \beta+48 c a_{1} a_{3} a_{4} \alpha \gamma+18 c a_{2} a_{3} a_{4} \alpha \beta=0$,

$\phi(\xi)^{-1}: 18 c a_{0} a_{3}^{2} \beta \gamma+36 c a_{0} a_{3} a_{4} \gamma^{2}+6 c a_{1} a_{3}^{2} \alpha \gamma$

$+6 c a_{2} a_{3} a_{4} \beta^{2}+18 c a_{2} a_{4}^{2} \beta \gamma-4 b a_{0} a_{3} \alpha \gamma$

$-12 b a_{0} a_{4} \beta \gamma-120 g a_{4} \alpha \beta \gamma^{2}-22 g a_{3} \alpha \beta^{2} \gamma$

$-720 z a_{3} \alpha^{2} \beta^{2} \gamma^{2}-114 z a_{3} \alpha \beta^{4} \gamma-3696 z a_{4} \alpha^{2} \beta \gamma^{3}$

$-2352 z a_{4} \alpha \beta^{3} \gamma^{2}-4 b a_{1} a_{4} \alpha \gamma+6 c a_{0}^{2} a_{3} \alpha \gamma$ 


$$
\begin{aligned}
& +18 c a_{0}^{2} a_{4} \beta \gamma+6 c a_{0} a_{1} a_{4} \beta^{2}-126 z a_{4} \beta^{5} \gamma \\
& -22 z a_{3} \alpha^{3} \gamma^{3}-30 g a_{4} \beta^{3} \gamma-16 g a_{3} \alpha^{2} \gamma^{2}+2 a_{3} \lambda^{2} \alpha \gamma \\
& +6 a_{4} \lambda^{2} \beta \gamma-2 b a_{0} a_{3} \beta^{2}-2 a a_{3} \alpha \gamma-6 a a_{4} \beta \gamma \\
& -2 b a_{1} a_{4} \beta^{2}-6 b a_{3}^{2} \beta \gamma-12 b a_{3} a_{4} \gamma^{2}+3 c a_{0}^{2} a_{3} \beta^{2} \\
& +18 c a_{1} a_{4}^{2} \gamma^{2}+3 c a_{1} a_{3}^{2} \beta^{2}-a a_{3} \beta^{2}+a_{3} \lambda^{2} \beta^{2} \\
& +6 c a_{3}^{3} \gamma^{2}-z a_{3} \beta^{6}-g a_{3} \beta^{4}+12 c a_{0} a_{1} a_{4} \alpha \gamma \\
& +36 c a_{1} a_{3} a_{4} \beta \gamma+12 c a_{2} a_{3} a_{4} \alpha \gamma=0 \text {, } \\
& \phi(\xi)^{0}: a_{3} \lambda^{2} \beta \gamma+a_{1} \lambda^{2} \beta \alpha+6 c a_{2}^{2} a_{4} \alpha^{2}+6 c a_{2} a_{4}^{2} \gamma^{2} \\
& +6 c a_{0} a_{1}^{2} \alpha^{2}+6 c a_{0} a_{3}^{2} \gamma^{2}+6 c a_{0}^{2} a_{4} \gamma^{2}+6 c a_{0}^{2} a_{2} \alpha^{2} \\
& -62 z a_{2} \beta^{4} \alpha^{2}-272 z a_{2} \gamma^{2} \alpha^{4}-z a_{1} \beta^{5} \alpha-62 z a_{4} \beta^{4} \gamma^{2} \\
& -22 z a_{4} \alpha^{2} \gamma^{4}-z a_{3} \beta^{5} \gamma-16 g a_{4} \alpha \gamma^{3}-14 g a_{4} \beta^{2} \gamma^{2} \\
& -g a_{3} \beta^{3} \gamma-g a_{1} \beta^{3} \alpha-14 g a_{2} \beta^{2} \alpha^{2}-16 g a_{2} \gamma \alpha^{3} \\
& -4 b a_{0} a_{4} \gamma^{2}-4 b a_{0} a_{2} \alpha^{2}-a a_{3} \beta \gamma-a a_{1} \beta \alpha \\
& -584 z a_{2} \beta^{2} \alpha^{3} \gamma-52 z a_{1} \beta^{3} \gamma \alpha^{2}-136 z a_{1} \beta \gamma^{2} \alpha^{3} \\
& -136 z a_{3} \alpha^{2} \beta \gamma^{3}-52 z a_{3} \alpha \beta^{3} \gamma^{2}-584 z a_{4} \alpha \beta^{2} \gamma^{3} \\
& -8 g a_{1} \beta \gamma \alpha^{2}-8 g a_{3} \alpha \beta \gamma^{2}-2 b a_{0} a_{3} \beta \gamma-2 b a_{0} a_{1} \beta \alpha \\
& -2 b a_{1} a_{4} \beta \gamma-2 b a_{2} a_{3} \alpha \beta+3 c a_{0}^{2} a_{3} \beta \gamma+3 c a_{0}^{2} a_{1} \beta \alpha \\
& +12 c a_{1} a_{3} a_{4} \gamma^{2}+3 c a_{1}^{2} a_{3} \alpha \beta+12 c a_{1} a_{2} a_{3} \alpha^{2} \\
& +3 c a_{1} a_{3}^{2} \beta \gamma-2 b a_{1}^{2} \alpha^{2}-2 b a_{3}^{2} \gamma^{2}-2 a a_{4} \gamma^{2}-2 a a_{2} \alpha^{2} \\
& +2 a_{4} \lambda^{2} \gamma^{2}+2 a_{2} \lambda^{2} \alpha^{2}+6 c a_{0} a_{1} a_{4} \beta \gamma+6 c a_{0} a_{2} a_{3} \alpha \beta \\
& +6 c a_{1} a_{2} a_{4} \alpha \beta+6 c a_{2} a_{3} a_{4} \beta \gamma=0, \\
& \phi(\xi): 18 c a_{2}^{2} a_{4} \alpha \beta-4 b a_{2} a_{3} \alpha \gamma-4 b a_{0} a_{1} \gamma \alpha+6 c a_{0}^{2} a_{1} \gamma \alpha \\
& +18 c a_{0}^{2} a_{2} \alpha \beta+6 c a_{0} a_{2} a_{3} \beta^{2}+18 c a_{0} a_{1}^{2} \alpha \beta \\
& +36 c a_{0} a_{1} a_{2} \alpha^{2}+6 c a_{1}^{2} a_{3} \alpha \gamma+6 c a_{1} a_{2} a_{4} \beta^{2} \\
& -12 b a_{0} a_{2} \alpha \beta-22 g a_{1} \beta^{2} \gamma \alpha-120 g a_{2} \beta \alpha^{2} \gamma \\
& -114 z a_{1} \beta^{4} \gamma \alpha-720 z a_{1} \beta^{2} \gamma^{2} \alpha^{2}-2352 z a_{2} \beta^{3} \gamma \alpha^{2} \\
& -3696 z a_{2} \beta \gamma^{2} \alpha^{3}-6 b a_{1}^{2} \alpha \beta-12 b a_{1} a_{2} \alpha^{2} \\
& -2 b a_{2} a_{3} \beta^{2}+3 c a_{0}^{2} a_{1} \beta^{2}+18 c a_{2}^{2} a_{3} \alpha^{2}+3 c a_{1}^{2} a_{3} \beta^{2} \\
& +2 a_{1} \lambda^{2} \gamma \alpha+6 a_{2} \lambda^{2} \alpha \beta-126 z a_{2} \beta^{5} \alpha-272 z a_{1} \gamma^{3} \alpha^{3} \\
& -16 g a_{1} \gamma^{2} \alpha^{2}-30 g a_{2} \beta^{3} \alpha-2 b a_{0} a_{1} \beta^{2}-2 a a_{1} \gamma \alpha \\
& -6 a a_{2} \alpha \beta+6 c a_{1}^{3} \alpha^{2}-z a_{1} \beta^{6}-g a_{1} \beta^{4}-a a_{1} \beta^{2}
\end{aligned}
$$

$$
\begin{aligned}
& +a_{1} \lambda^{2} \beta^{2}+12 c a_{0} a_{2} a_{3} \alpha \gamma+36 c a_{1} a_{2} a_{3} \alpha \beta \\
& +12 c a_{1} a_{2} a_{4} \alpha \gamma=0,
\end{aligned}
$$$$
\phi(\xi)^{2}: 24 c a_{1} a_{2} a_{3} \beta^{2}+9 c a_{1}^{2} a_{3} \beta \gamma+24 c a_{2}^{2} a_{4} \alpha \gamma
$$$$
-16 b a_{0} a_{2} \alpha \gamma-60 g a_{1} \beta \alpha \gamma^{2}-232 g a_{2} \beta^{2} \alpha \gamma
$$$$
-1848 z a_{1} \beta \gamma^{3} \alpha^{2}-1176 z a_{1} \beta^{3} \alpha \gamma^{2}-3096 z a_{2} \beta^{4} \gamma \alpha
$$$$
-13320 z a_{2} \beta^{2} \gamma^{2} \alpha^{2}-30 b a_{1} a_{2} \alpha \beta-6 b a_{2} a_{3} \beta \gamma
$$$$
-6 b a_{0} a_{1} \beta \gamma+9 c a_{0}^{2} a_{1} \beta \gamma+24 c a_{0}^{2} a_{2} \alpha \gamma+45 c a_{2}^{2} a_{3} \alpha \beta
$$$$
+24 c a_{0} a_{1}^{2} \alpha \gamma-3968 z a_{2} \gamma^{3} \alpha^{3}-63 z a_{1} \beta^{5} \gamma
$$$$
-15 g a_{1} \beta^{3} \gamma-136 g a_{2} \gamma^{2} \alpha^{2}+3 a_{1} \lambda^{2} \beta \gamma+8 a_{2} \lambda^{2} \alpha \gamma
$$$$
-8 b a_{0} a_{2} \beta^{2}-3 a a_{1} \beta \gamma-8 a a_{2} \alpha \gamma-8 b a_{1}^{2} \alpha \gamma
$$$$
+12 c a_{0}^{2} a_{2} \beta^{2}+36 c a_{0} a_{2}^{2} \alpha^{2}+12 c a_{0} a_{1}^{2} \beta^{2}+15 c a_{1}^{3} \alpha \beta
$$$$
+36 c a_{1}^{2} a_{2} \alpha^{2}+12 c a_{2}^{2} a_{4} \beta^{2}-4 a a_{2} \beta^{2}+4 a_{2} \lambda^{2} \beta^{2}
$$$$
-12 b a_{2}^{2} \alpha^{2}-4 b a_{1}^{2} \beta^{2}-64 z a_{2} \beta^{6}-16 g a_{2} \beta^{4}
$$$$
+90 c a_{0} a_{1} a_{2} \alpha \beta+18 c a_{0} a_{2} a_{3} \beta \gamma+48 c a_{1} a_{2} a_{3} \alpha \gamma
$$$$
+18 c a_{1} a_{2} a_{4} \beta \gamma=0 \text {, }
$$$$
\phi(\xi)^{3}: 6 c a_{1}^{2} a_{3} \gamma^{2}+60 c a_{1} a_{2}^{2} \alpha^{2}+10 a_{2} \lambda^{2} \beta \gamma
$$$$
-1330 z a_{2} \beta^{5} \gamma-602 z a_{1} \beta^{4} \gamma^{2}-1232 z a_{1} \gamma^{4} \alpha^{2}
$$$$
-50 g a_{1} \beta^{2} \gamma^{2}-40 g a_{1} \alpha \gamma^{3}-130 g a_{2} \beta^{3} \gamma-4 b a_{0} a_{1} \gamma
$$$$
-10 a a_{2} \beta \gamma-10 b a_{1}^{2} \beta \gamma-18 b a_{1} a_{2} \beta^{2}-28 b a_{2}^{2} \alpha \beta
$$$$
-4 b a_{2} a_{3} \gamma^{2}+6 c a_{0}^{2} a_{1} \gamma^{2}+27 c a_{2}^{2} a_{3} \beta^{2}+18 c a_{1}^{3} \alpha \gamma
$$$$
+108 c a_{0} a_{1} a_{2} \alpha \gamma+60 c a_{1} a_{2} a_{3} \beta \gamma-20 b a_{0} a_{2} \beta \gamma
$$$$
-440 g a_{2} \beta \alpha \gamma^{2}-3584 z a_{1} \beta^{2} \alpha \gamma^{3}-22960 z a_{2} \beta \gamma^{3} \alpha^{2}
$$$$
+12 c a_{1} a_{2} a_{4} \gamma^{2}+84 c a_{1}^{2} a_{2} \alpha \beta+30 c a_{2}^{2} a_{4} \beta \gamma
$$$$
-17920 z a_{2} \beta^{3} \alpha \gamma^{2}+54 c a_{2}^{2} a_{3} \alpha \gamma-36 b a_{1} a_{2} \alpha \gamma
$$$$
+30 c a_{0}^{2} a_{2} \beta \gamma+30 c a_{0} a_{1}^{2} \beta \gamma+54 c a_{0} a_{1} a_{2} \beta^{2}
$$$$
+84 c a_{0} a_{2}^{2} \alpha \beta+12 c a_{0} a_{2} a_{3} \gamma^{2}-2 a a_{1} \gamma^{2}+2 a_{1} \lambda^{2} \gamma^{2}
$$$$
+9 c a_{1}^{3} \beta^{2}=0
$$

$\phi(\xi)^{4}: 135 c a_{1} a_{2}^{2} \alpha \beta+36 c a_{1} a_{2} a_{3} \gamma^{2}+96 c a_{1}^{2} a_{2} \alpha \gamma$

$-4200 z a_{1} \beta \gamma^{4} \alpha-40152 z a_{2} \beta^{2} \alpha \gamma^{3}+63 c a_{2}^{2} a_{3} \beta \gamma$ 


$$
\begin{aligned}
& -42 b a_{1} a_{2} \beta \gamma+96 c a_{0} a_{2}^{2} \alpha \gamma-8106 z a_{2} \beta^{4} \gamma^{2} \\
& -12096 z a_{2} \gamma^{4} \alpha^{2}-2100 z a_{1} \beta^{3} \gamma^{3}-60 g a_{1} \beta \gamma^{3} \\
& -330 g a_{2} \beta^{2} \gamma^{2}-240 g a_{2} \gamma^{3} \alpha-12 b a_{0} a_{2} \gamma^{2} \\
& -32 b a_{2}^{2} \alpha \gamma+18 c a_{0}^{2} a_{2} \gamma^{2}+18 c a_{0} a_{1}^{2} \gamma^{2}+48 c a_{0} a_{2}^{2} \beta^{2} \\
& +21 c a_{1}^{3} \beta \gamma+48 c a_{1}^{2} a_{2} \beta^{2}+18 c a_{2}^{2} a_{4} \gamma^{2}-6 a a_{2} \gamma^{2} \\
& -6 a a_{2} \gamma^{2}+6 a_{2} \lambda^{2} \gamma^{2}+30 c a_{2}^{3} \alpha^{2}-6 b a_{1}^{2} \gamma^{2} \\
& -16 b a_{2}^{2} \beta^{2}+126 c a_{0} a_{1} a_{2} \beta \gamma=0, \\
\phi & (\xi)^{5}:-336 g a_{2} \beta \gamma^{3}-24 b a_{1} a_{2} \gamma^{2}-36 b a_{2}^{2} \beta \gamma \\
& +36 c a_{2}^{2} a_{3} \gamma^{2}+75 c a_{1} a_{2}^{2} \beta^{2}+66 c a_{2}^{3} \alpha \beta \\
& -21840 z a_{2} \beta^{3} \gamma^{3}-3360 z a_{1} \beta^{2} \gamma^{4}-1680 z a_{1} \gamma^{5} \alpha \\
& +72 c a_{0} a_{1} a_{2} \gamma^{2}+108 c a_{0} a_{2}^{2} \beta \gamma+150 c a_{1} a_{2}^{2} \alpha \gamma \\
& +108 c a_{1}^{2} a_{2} \beta \gamma-38640 z a_{2} \beta \gamma^{4} \alpha+12 c a_{1}^{3} \gamma^{2}
\end{aligned}
$$

$$
\begin{aligned}
& -24 g a_{1} \gamma^{4}=0, \\
& \phi(\xi)^{6}: 60 c a_{0} a_{2}^{2} \gamma^{2}+60 c a_{1}^{2} a_{2} \gamma^{2}+72 c a_{2}^{3} \alpha \gamma \\
& -29400 z a_{2} \beta^{2} \gamma^{2}-13440 z a_{2} \gamma^{5} \alpha-2520 z a_{1} \beta \gamma^{5} \\
& +165 c a_{1} a_{2}^{2} \beta \gamma+36 c a_{2}^{3} \beta^{2}-20 b a_{2}^{2} \gamma^{2} \\
& \quad-120 g a_{2} \gamma^{4}=0, \\
& \phi(\xi)^{7}:-19440 \beta \gamma^{5} z a_{2}-720 a_{1} z \gamma^{6}+8 c a_{2}^{3} \beta \gamma \\
& \quad+90 c a_{1} a_{2}^{2} \gamma^{2}=0, \\
& \phi(\xi)^{8}:-5040 a_{2} z \gamma^{6}+42 c a_{2}^{3} \gamma^{2}=0 .
\end{aligned}
$$

Solving the system of algebraic equations with the aid of Maple, using (18), we obtain the following results.

First Set

$$
\begin{aligned}
& a_{0}= \pm \frac{1}{30}\left(\frac{40 \sqrt{30} \sqrt{h c} \gamma \alpha h+5 \sqrt{30} \sqrt{h c} \beta^{2} h+\sqrt{30} \sqrt{h c} g+10 b h}{h c}\right), \\
& a_{1}=0 \\
& a_{2}=0, \\
& a_{3}= \pm \frac{2 \beta \sqrt{30} \sqrt{h c} \alpha}{c}, \\
& a_{4}= \pm \frac{2 \sqrt{30} \sqrt{h c} \alpha^{2}}{c}, \\
& \lambda= \pm \frac{1}{30} \frac{\sqrt{-30 c h\left(720 \alpha^{2} \gamma^{2} c h^{2}-360 \alpha \gamma \beta^{2} c h^{2}+45 \beta^{4} c h^{2}-30 a c h-10 b^{2} h+3 c g^{2}\right)}}{c h} .
\end{aligned}
$$

Case 1. When $\beta=0, \alpha>0$, and $\gamma>0, \phi(\xi)=(\sqrt{\alpha \gamma} /$ where $\gamma) \tan (\sqrt{\alpha \gamma} \xi)$, the periodic solutions of (1) are

$$
\begin{array}{r}
V_{1}(x, t) \\
=\frac{1}{30}\left(\frac{40 \sqrt{30} \sqrt{h c} \gamma \alpha h+\sqrt{30} \sqrt{h c} g+10 b h}{h c}\right) \\
\quad+\frac{2 \sqrt{30} \sqrt{h c} \gamma \alpha^{2}}{c} \cot ^{2}\left(\sqrt{\alpha \gamma}\left(x+\lambda t+\xi_{0}\right)\right), \\
V_{2}(x, t) \\
=\frac{1}{30}\left(\frac{40 \sqrt{30} \sqrt{h c} \gamma \alpha h+\sqrt{30} \sqrt{h c} g+10 b h}{h c}\right) \\
\quad+\frac{2 \sqrt{30} \sqrt{h c} \gamma \alpha^{2}}{c} \cot ^{2}\left(\sqrt{\alpha \gamma}\left(x-\lambda t+\xi_{0}\right)\right),
\end{array}
$$

$$
\begin{aligned}
\lambda= & \frac{1}{30} \\
& \cdot \frac{\sqrt{-30 c h\left(720 \alpha^{2} \gamma^{2} c h^{2}-30 a c h-10 b^{2} h+3 c g^{2}\right)}}{c h} .
\end{aligned}
$$

Case 2. When $\beta=0, \alpha<0$, and $\gamma>0, \phi(\xi)=$ $(\sqrt{-\alpha \gamma} / \gamma) \tanh (-\sqrt{-\alpha \gamma} \xi)$, the periodic solutions of (1) are

$$
\begin{aligned}
V_{3}(x, t) & \\
= & \frac{1}{30}\left(\frac{40 \sqrt{30} \sqrt{h c} \gamma \alpha h+\sqrt{30} \sqrt{h c} g+10 b h}{h c}\right) \\
& -\frac{2 \sqrt{30} \sqrt{h c} \alpha \gamma}{c} \operatorname{coth}^{2}\left(-\sqrt{-\alpha \gamma}\left(x+\lambda t+\xi_{0}\right)\right),
\end{aligned}
$$




$$
\begin{aligned}
V_{4}(x, t) & \\
= & \frac{1}{30}\left(\frac{40 \sqrt{30} \sqrt{h c} \gamma \alpha h+\sqrt{30} \sqrt{h c} g+10 b h}{h c}\right) \\
& -\frac{2 \sqrt{30} \sqrt{h c} \alpha \gamma}{c} \operatorname{coth}^{2}\left(-\sqrt{-\alpha \gamma}\left(x-\lambda t+\xi_{0}\right)\right),
\end{aligned}
$$

where

$$
\begin{aligned}
\lambda= & \frac{1}{30} \\
& \cdot \frac{\sqrt{-30 c h\left(720 \alpha^{2} \gamma^{2} c h^{2}-30 a c h-10 b^{2} h+3 c g^{2}\right)}}{c h} .
\end{aligned}
$$

Case 3. When $\gamma=0, \phi(\xi)=\left(-\alpha+\beta e^{\beta \xi}\right) / \beta$, the combined formal single kink solutions of (1) are

$$
\begin{aligned}
V_{5}(x, t)= & \frac{1}{30}\left(\frac{5 \sqrt{30} \sqrt{h c} \beta^{2} h+\sqrt{30} \sqrt{h c} g+10 b h}{h c}\right) \\
& +\frac{2 \beta^{2} \sqrt{30} \sqrt{h c} \alpha}{c\left(-\alpha+\beta e^{\beta\left(x+\lambda t+\xi_{0}\right)}\right)} \\
& +\frac{2 \beta^{2} \sqrt{30} \sqrt{h c} \alpha^{2}}{c\left(-\alpha+\beta e^{\beta\left(x+\lambda t+\xi_{0}\right)}\right)^{2}}, \\
V_{6}(x, t)= & \frac{1}{30}\left(\frac{5 \sqrt{30} \sqrt{h c} \beta^{2} h+\sqrt{30} \sqrt{h c} g+10 b h}{h c}\right) \\
& +\frac{2 \beta^{2} \sqrt{30} \sqrt{h c} \alpha}{c\left(-\alpha+\beta e^{\beta\left(x-\lambda t+\xi_{0}\right)}\right)} \\
& +\frac{2 \beta^{2} \sqrt{30} \sqrt{h c} \alpha^{2}}{c\left(-\alpha+\beta e^{\beta\left(x-\lambda t+\xi_{0}\right)}\right)^{2}},
\end{aligned}
$$

where

$$
\lambda=\frac{1}{30} \frac{\sqrt{-30 c h\left(45 \beta^{4} c h^{2}-30 a c h-10 b^{2} h+3 c g^{2}\right)}}{c h} .
$$

Case 4. When $\beta^{2} \neq 0$ and $\beta^{2}=4 \alpha \gamma, \phi(\xi)=-2 \alpha(\beta \xi+2) / \beta^{2} \xi$, the rational solutions of (1) are

$$
\begin{aligned}
V_{7}(x, t) & \frac{1}{30}\left(\frac{15 \sqrt{30} \sqrt{h c} \beta^{2} h+\sqrt{30} \sqrt{h c} g+10 b h}{c h}\right) \\
& -\frac{\beta^{3} \sqrt{30} \sqrt{h c}\left(x-\lambda t+\xi_{0}\right)}{c\left(\beta\left(x-\lambda t+\xi_{0}\right)+2\right)} \\
& +\frac{\beta^{5} \sqrt{30} \sqrt{h c}\left(x-\lambda t+\xi_{0}\right)^{2}}{2 c\left(\beta\left(x-\lambda t+\xi_{0}\right)+2\right)^{2}}, \\
V_{8}(x, & t) \\
= & \frac{1}{30}\left(\frac{15 \sqrt{30} \sqrt{h c} \beta^{2} h+\sqrt{30} \sqrt{h c} g+10 b h}{c h}\right) \\
& -\frac{\beta^{3} \sqrt{30} \sqrt{h c}\left(x+\lambda t+\xi_{0}\right)}{c\left(\beta\left(x+\lambda t+\xi_{0}\right)+2\right)} \\
& +\frac{\beta^{5} \sqrt{30} \sqrt{h c}\left(x+\lambda t+\xi_{0}\right)^{2}}{2 c\left(\beta\left(x+\lambda t+\xi_{0}\right)+2\right)^{2}},
\end{aligned}
$$

where

$$
\lambda=\frac{1}{30} \frac{\sqrt{-30 c h\left(-30 a c h-10 b^{2} h+3 c g^{2}\right)}}{c h} .
$$

Case 5. When $\beta^{2}<4 \alpha \gamma$ and $\gamma \neq 0, \phi(\xi)=$ $\left(\sqrt{4 \alpha \gamma-\beta^{2}} \tan \left((1 / 2) \sqrt{4 \alpha \gamma-\beta^{2}} \xi\right)-\beta\right) / 2 \gamma$, the periodic solutions of (1) are

$$
\begin{aligned}
V_{9}(x, t)= & \frac{1}{30}\left(\frac{40 \sqrt{30} \sqrt{h c} \gamma \alpha h+5 \sqrt{30} \sqrt{h c} \beta^{2} h+\sqrt{30} \sqrt{h c} g+10 b h}{h c}\right) \\
& +\frac{2 \beta \sqrt{30} \sqrt{h c} \alpha}{c}\left(\frac{2 \gamma}{\sqrt{4 \alpha \gamma-\beta^{2}} \tan \left((1 / 2) \sqrt{4 \alpha \gamma-\beta^{2}}\left(x+\lambda t+\xi_{0}\right)\right)-\beta}\right) \\
& +\frac{2 \sqrt{30} \sqrt{h c} \alpha^{2}}{c}\left(\frac{4 \gamma^{2}}{\left(\sqrt{4 \alpha \gamma-\beta^{2}} \tan \left((1 / 2) \sqrt{4 \alpha \gamma-\beta^{2}}\left(x+\lambda t+\xi_{0}\right)\right)-\beta\right)^{2}}\right), \\
V_{10}(x, t)= & \frac{1}{30}\left(\frac{40 \sqrt{30} \sqrt{h c} \gamma \alpha h+5 \sqrt{30} \sqrt{h c} \beta^{2} h+\sqrt{30} \sqrt{h c} g+10 b h}{h c}\right)
\end{aligned}
$$




$$
\begin{aligned}
& +\frac{2 \beta \sqrt{30} \sqrt{h c} \alpha}{c}\left(\frac{2 \gamma}{\sqrt{4 \alpha \gamma-\beta^{2}} \tan \left((1 / 2) \sqrt{4 \alpha \gamma-\beta^{2}}\left(x-\lambda t+\xi_{0}\right)\right)-\beta}\right) \\
& +\frac{2 \sqrt{30} \sqrt{h c} \alpha^{2}}{c}\left(\frac{4 \gamma^{2}}{\left(\sqrt{4 \alpha \gamma-\beta^{2}} \tan \left((1 / 2) \sqrt{4 \alpha \gamma-\beta^{2}}\left(x-\lambda t+\xi_{0}\right)\right)-\beta\right)^{2}}\right),
\end{aligned}
$$

where

$$
\lambda=\frac{1}{30} \frac{\sqrt{-30 c h\left(720 \alpha^{2} \gamma^{2} c h^{2}-360 \alpha \gamma \beta^{2} c h^{2}+45 \beta^{4} c h^{2}-30 a c h-10 b^{2} h+3 c g^{2}\right)}}{c h} .
$$

Case 6. When $\beta^{2}>4 \alpha \gamma$ and $\gamma \neq 0, \phi(\xi)=$ $\left(\sqrt{\beta^{2}-4 \alpha \gamma} \tanh \left((1 / 2) \sqrt{\beta^{2}-4 \alpha \gamma} \xi\right)-\beta\right) / 2 \gamma$, the periodic solutions of (1) are

$$
\begin{aligned}
V_{11}(x, t)= & \frac{1}{30}\left(\frac{40 \sqrt{30} \sqrt{h c} \gamma \alpha h+5 \sqrt{30} \sqrt{h c} \beta^{2} h+\sqrt{30} \sqrt{h c} g+10 b h}{h c}\right) \\
& +\frac{2 \beta \sqrt{30} \sqrt{h c} \alpha}{c}\left(\frac{2 \gamma}{\sqrt{\beta^{2}-4 \alpha \gamma} \tanh \left((1 / 2) \sqrt{\beta^{2}-4 \alpha \gamma}\left(x+\lambda t+\xi_{0}\right)\right)-\beta}\right) \\
& +\frac{2 \sqrt{30} \sqrt{h c} \alpha^{2}}{c}\left(\frac{4 \gamma^{2}}{\left(\sqrt{\beta^{2}-4 \alpha \gamma} \tanh \left((1 / 2) \sqrt{\beta^{2}-4 \alpha \gamma}\left(x+\lambda t+\xi_{0}\right)\right)-\beta\right)^{2}}\right), \\
V_{12}(x, t)= & \frac{1}{30}\left(\frac{40 \sqrt{30} \sqrt{h c} \gamma \alpha h+5 \sqrt{30} \sqrt{h c} \beta^{2} h+\sqrt{30} \sqrt{h c} g+10 b h}{h c}\right) \\
& +\frac{2 \beta \sqrt{30} \sqrt{h c} \alpha}{c}\left(\frac{2 \gamma}{\sqrt{\beta^{2}-4 \alpha \gamma} \tanh \left((1 / 2) \sqrt{\beta^{2}-4 \alpha \gamma}\left(x-\lambda t+\xi_{0}\right)\right)-\beta}\right) \\
& +\frac{2 \sqrt{30} \sqrt{h c} \alpha^{2}}{c}\left(\frac{4 \gamma^{2}}{\left(\sqrt{\beta^{2}-4 \alpha \gamma} \tanh \left((1 / 2) \sqrt{\beta^{2}-4 \alpha \gamma}\left(x-\lambda t+\xi_{0}\right)\right)-\beta\right)^{2}}\right),
\end{aligned}
$$

where

$$
\lambda=\frac{1}{30} \frac{\sqrt{-30 c h\left(720 \alpha^{2} \gamma^{2} c h^{2}-360 \alpha \gamma \beta^{2} c h^{2}+45 \beta^{4} c h^{2}-30 a c h-10 b^{2} h+3 c g^{2}\right)}}{c h} .
$$


Second Set

$$
\begin{aligned}
& a_{0}= \pm \frac{1}{30}\left(\frac{40 \sqrt{30} \sqrt{h c} \gamma \alpha h+5 \sqrt{30} \sqrt{h c} \beta^{2} h+\sqrt{30} \sqrt{h c} g+10 b h}{h c}\right), \\
& a_{1}= \pm \frac{2 \beta \sqrt{30} \sqrt{h c} \gamma}{c}, \\
& a_{2}= \pm \frac{2 \sqrt{30} \sqrt{h c} \gamma^{2}}{c}, \\
& a_{3}=0, \\
& a_{4}=0, \\
& \lambda= \pm \frac{1}{30} \frac{\sqrt{-30 c h\left(720 \alpha^{2} \gamma^{2} c h^{2}-360 \alpha \gamma \beta^{2} c h^{2}+45 \beta^{4} c h^{2}-30 a c h-10 b^{2} h+3 c g^{2}\right)}}{c h} .
\end{aligned}
$$

Case 1. When $\alpha=0, \phi(\xi)=\beta /\left(-\gamma+\beta e^{-\beta \xi}\right)$, the combined formal single kink solutions of (1) are

$$
\begin{aligned}
V_{13}(x, t)= & \frac{1}{30}\left(\frac{5 \sqrt{30} \sqrt{h c} \beta^{2} h+\sqrt{30} \sqrt{h c} g+10 b h}{h c}\right) \\
& +\frac{2 \beta^{2} \sqrt{30} \sqrt{h c} \gamma}{c\left(-\gamma+\beta e^{-\beta\left(x+\lambda t+\xi_{0}\right)}\right)} \\
& +\frac{2 \beta^{2} \sqrt{30} \sqrt{h c} \gamma^{2}}{c\left(-\gamma+\beta e^{-\beta\left(x+\lambda t+\xi_{0}\right)}\right)^{2}}, \\
V_{14}(x, t)= & \frac{1}{30}\left(\frac{5 \sqrt{30} \sqrt{h c} \beta^{2} h+\sqrt{30} \sqrt{h c} g+10 b h}{h c}\right) \\
& +\frac{2 \beta^{2} \sqrt{30} \sqrt{h c} \gamma}{c\left(-\gamma+\beta e^{-\beta\left(x-\lambda t+\xi_{0}\right)}\right)} \\
& +\frac{2 \beta^{2} \sqrt{30} \sqrt{h c} \gamma^{2}}{c\left(-\gamma+\beta e^{-\beta\left(x-\lambda t+\xi_{0}\right)}\right)^{2}},
\end{aligned}
$$

where

$$
\lambda=\frac{1}{30} \frac{\sqrt{-30 c h\left(45 \beta^{4} c h^{2}-30 a c h-10 b^{2} h+3 c g^{2}\right)}}{c h} .
$$

Case 2. When $\beta=0, \alpha>0$, and $\gamma>0, \phi(\xi)=$ $(\sqrt{\alpha \gamma} / \gamma) \tan (\sqrt{\alpha \gamma} \xi)$, the periodic solutions of (1) are

$$
\begin{aligned}
& V_{15}(x, t) \\
& =\frac{1}{30}\left(\frac{40 \sqrt{30} \sqrt{h c} \gamma \alpha h+\sqrt{30} \sqrt{h c} g+10 b h}{h c}\right) \\
& \quad+\frac{2 \sqrt{30} \sqrt{h c} \alpha \gamma}{c} \tan ^{2}\left(\sqrt{\alpha \gamma}\left(x+\lambda t+\xi_{0}\right)\right),
\end{aligned}
$$

$$
\begin{aligned}
& V_{16}(x, t) \\
& =\frac{1}{30}\left(\frac{40 \sqrt{30} \sqrt{h c} \gamma \alpha h+\sqrt{30} \sqrt{h c} g+10 b h}{h c}\right) \\
& \quad+\frac{2 \sqrt{30} \sqrt{h c} \alpha \gamma}{c} \tan ^{2}\left(\sqrt{\alpha \gamma}\left(x-\lambda t+\xi_{0}\right)\right),
\end{aligned}
$$

where

$$
\begin{aligned}
\lambda= & \frac{1}{30} \\
& \frac{\sqrt{-30 c h\left(720 \alpha^{2} \gamma^{2} c h^{2}-30 a c h-10 b^{2} h+3 c g^{2}\right)}}{c h} .
\end{aligned}
$$

Case 3. When $\beta=0, \alpha<0$, and $\gamma>0, \phi(\xi)=$ $(\sqrt{-\alpha \gamma} / \gamma) \tanh (-\sqrt{-\alpha \gamma} \xi)$, the periodic solutions of (1) are

$$
\begin{aligned}
V_{17} & (x, t) \\
= & \frac{1}{30}\left(\frac{40 \sqrt{30} \sqrt{h c} \gamma \alpha h+\sqrt{30} \sqrt{h c} g+10 b h}{h c}\right) \\
+ & \frac{2 \sqrt{30} \sqrt{h c}(\sqrt{-\alpha \gamma})^{2}}{c} \\
\cdot & \tanh ^{2}\left(-\sqrt{-\alpha \gamma}\left(x+\lambda t+\xi_{0}\right)\right), \\
V_{18} & (x, t) \\
= & \frac{1}{30}\left(\frac{40 \sqrt{30} \sqrt{h c} \gamma \alpha h+\sqrt{30} \sqrt{h c} g+10 b h}{h c}\right) \\
+ & \frac{2 \sqrt{30} \sqrt{h c}(\sqrt{-\alpha \gamma})^{2}}{c} \\
\cdot & \tanh ^{2}\left(-\sqrt{-\alpha \gamma}\left(x-\lambda t+\xi_{0}\right)\right),
\end{aligned}
$$


where

$$
\begin{aligned}
\lambda= & \frac{1}{30} \\
& \cdot \frac{\sqrt{-30 c h\left(720 \alpha^{2} \gamma^{2} c h^{2}-30 a c h-10 b^{2} h+3 c g^{2}\right)}}{c h} .
\end{aligned}
$$

Case 4. When $\alpha=\beta=0, \phi(\xi)=-1 / \gamma \xi$, the rational solutions of (1) are

$$
\begin{aligned}
& V_{19}(x, t)=\frac{1}{30}\left(\frac{\sqrt{30} \sqrt{h c} g+10 b h}{h c}\right) \\
& +\frac{2 \sqrt{30} \sqrt{h c} \gamma^{2}}{c\left(\gamma\left(x+\lambda t+\xi_{0}\right)\right)^{2}}, \\
& V_{20}(x, t)=\frac{1}{30}\left(\frac{\sqrt{30} \sqrt{h c} g+10 b h}{h c}\right) \\
& +\frac{2 \sqrt{30} \sqrt{h c} \gamma^{2}}{c\left(\gamma\left(x-\lambda t+\xi_{0}\right)\right)^{2}}, \\
& V_{21}(x, t)=\frac{1}{30}\left(\frac{\sqrt{30} \sqrt{h c} g+10 b h}{h c}\right) \\
& -\frac{2 \sqrt{30} \sqrt{h c} \gamma^{2}}{c\left(\gamma\left(x+\lambda t+\xi_{0}\right)\right)^{2}} \\
& V_{22}(x, t)=\frac{1}{30}\left(\frac{\sqrt{30} \sqrt{h c} g+10 b h}{h c}\right) \\
& -\frac{2 \sqrt{30} \sqrt{h c} \gamma^{2}}{c\left(\gamma\left(x-\lambda t+\xi_{0}\right)\right)^{2}}, \\
& V_{23}(x, t)=-\frac{1}{30}\left(\frac{\sqrt{30} \sqrt{h c} g+10 b h}{h c}\right) \\
& +\frac{2 \sqrt{30} \sqrt{h c} \gamma^{2}}{c\left(\gamma\left(x+\lambda t+\xi_{0}\right)\right)^{2}}, \\
& V_{24}(x, t)=-\frac{1}{30}\left(\frac{\sqrt{30} \sqrt{h c} g+10 b h}{h c}\right) \\
& +\frac{2 \sqrt{30} \sqrt{h c} \gamma^{2}}{c\left(\gamma\left(x-\lambda t+\xi_{0}\right)\right)^{2}}, \\
& V_{25}(x, t)=-\frac{1}{30}\left(\frac{\sqrt{30} \sqrt{h c} g+10 b h}{h c}\right) \\
& -\frac{2 \sqrt{30} \sqrt{h c} \gamma^{2}}{c\left(\gamma\left(x+\lambda t+\xi_{0}\right)\right)^{2}},
\end{aligned}
$$

$$
\begin{aligned}
V_{26}(x, t)= & -\frac{1}{30}\left(\frac{\sqrt{30} \sqrt{h c} g+10 b h}{h c}\right) \\
& -\frac{2 \sqrt{30} \sqrt{h c} \gamma^{2}}{c\left(\gamma\left(x-\lambda t+\xi_{0}\right)\right)^{2}},
\end{aligned}
$$

where

$$
\lambda=\frac{1}{30} \frac{\sqrt{-30 c h\left(-30 a c h-10 b^{2} h+3 c g^{2}\right)}}{c h} .
$$

Case 5. When $\beta^{2} \neq 0$ and $\beta^{2}=4 \alpha \gamma, \phi(\xi)=-2 \alpha(\beta \xi+2) / \beta^{2} \xi$, the rational solutions of (1) are

$$
\begin{aligned}
V_{27}(x, t)= & \frac{1}{30} \frac{15 \sqrt{30} \sqrt{h c} \beta^{2} h+10 b h+\sqrt{30} \sqrt{c h} g}{c h} \\
& -\frac{\beta \sqrt{30} \sqrt{c h}\left(\beta\left(x-\lambda t+\xi_{0}\right)+2\right)}{c\left(x-\lambda t+\xi_{0}\right)} \\
& +\frac{4 \beta \sqrt{30} \sqrt{c h}\left(\beta\left(x-\lambda t+\xi_{0}\right)+2\right)^{2}}{c\left(x-\lambda t+\xi_{0}\right)^{2}},
\end{aligned}
$$$$
V_{28}(x, t)=\frac{1}{30} \frac{15 \sqrt{30} \sqrt{h c} \beta^{2} h+10 b h+\sqrt{30} \sqrt{c h} g}{c h}
$$$$
-\frac{\beta \sqrt{30} \sqrt{c h}\left(\beta\left(x+\lambda t+\xi_{0}\right)+2\right)}{c\left(x+\lambda t+\xi_{0}\right)}
$$$$
+\frac{4 \beta \sqrt{30} \sqrt{c h}\left(\beta\left(x+\lambda t+\xi_{0}\right)+2\right)^{2}}{c\left(x+\lambda t+\xi_{0}\right)^{2}},
$$

where

$$
\lambda=\frac{1}{30} \frac{\sqrt{-30 c h\left(-30 a c h-10 b^{2} h+3 c g^{2}\right)}}{c h} .
$$

Case 6. When $\beta^{2}<4 \alpha \gamma$ and $\gamma \neq 0, \phi(\xi)=$ $\left(\sqrt{4 \alpha \gamma-\beta^{2}} \tan \left((1 / 2) \sqrt{4 \alpha \gamma-\beta^{2}} \xi\right)-\beta\right) / 2 \gamma$, the periodic solutions of (1) are

$$
\begin{aligned}
& V_{29}(x, t) \\
& =\frac{1}{30}\left(\frac{40 \sqrt{30} \sqrt{h c} \gamma \alpha h+5 \sqrt{30} \sqrt{h c} \beta^{2} h+\sqrt{30} \sqrt{h c} g+10 b h}{h c}\right) \\
& +\frac{\beta \sqrt{30} \sqrt{h c}}{c}\left(\sqrt{4 \alpha \gamma-\beta^{2}} \tan \left(\frac{1}{2} \sqrt{4 \alpha \gamma-\beta^{2}}\left(x+\lambda t+\xi_{0}\right)\right)\right.
\end{aligned}
$$




$$
\begin{aligned}
& -\beta) \\
& +\frac{\sqrt{30} \sqrt{h c}}{2 c}\left(\sqrt{4 \alpha \gamma-\beta^{2}} \tan \left(\frac{1}{2} \sqrt{4 \alpha \gamma-\beta^{2}}\left(x+\lambda t+\xi_{0}\right)\right)\right. \\
& -\beta)^{2} \\
& V_{30}(x, t) \\
& =\frac{1}{30}\left(\frac{40 \sqrt{30} \sqrt{h c} \gamma \alpha h+5 \sqrt{30} \sqrt{h c} \beta^{2} h+\sqrt{30} \sqrt{h c} g+10 b h}{h c}\right) \\
& V_{31}(x, t) \\
& =\frac{1}{30}\left(\frac{40 \sqrt{30} \sqrt{h c} \gamma \alpha h+5 \sqrt{30} \sqrt{h c} \beta^{2} h+\sqrt{30} \sqrt{h c} g+10 b h}{h c}\right) \\
& +\frac{\beta \sqrt{30} \sqrt{h c}}{c}\left(\sqrt{\beta^{2}-4 \alpha \gamma} \tanh \left(\frac{1}{2} \sqrt{\beta^{2}-4 \alpha \gamma}\left(x+\lambda t+\xi_{0}\right)\right)\right. \\
& -\beta) \\
& +\frac{\sqrt{30} \sqrt{h c}}{2 c}\left(\sqrt{\beta^{2}-4 \alpha \gamma} \tanh \left(\frac{1}{2} \sqrt{\beta^{2}-4 \alpha \gamma}\left(x+\lambda t+\xi_{0}\right)\right)\right. \\
& -\beta)^{2}
\end{aligned}
$$$$
+\frac{\beta \sqrt{30} \sqrt{h c}}{c}\left(\sqrt{4 \alpha \gamma-\beta^{2}} \tan \left(\frac{1}{2} \sqrt{4 \alpha \gamma-\beta^{2}}\left(x-\lambda t+\xi_{0}\right)\right)\right.
$$$$
-\beta)
$$$$
+\frac{\sqrt{30} \sqrt{h c}}{2 c}\left(\sqrt{4 \alpha \gamma-\beta^{2}} \tan \left(\frac{1}{2} \sqrt{4 \alpha \gamma-\beta^{2}}\left(x-\lambda t+\xi_{0}\right)\right)\right.
$$$$
-\beta)^{2}
$$

where

$$
\lambda=\frac{1}{30} \frac{\sqrt{-30 c h\left(720 \alpha^{2} \gamma^{2} c h^{2}-360 \alpha \gamma \beta^{2} c h^{2}+45 \beta^{4} c h^{2}-30 a c h-10 b^{2} h+3 c g^{2}\right)}}{c h} .
$$

$V_{32}(x, t)$

$$
\begin{aligned}
& =\frac{1}{30}\left(\frac{40 \sqrt{30} \sqrt{h c} \gamma \alpha h+5 \sqrt{30} \sqrt{h c} \beta^{2} h+\sqrt{30} \sqrt{h c} g+10 b h}{h c}\right) \\
& +\frac{\beta \sqrt{30} \sqrt{h c}}{c}\left(\sqrt{\beta^{2}-4 \alpha \gamma} \tanh \left(\frac{1}{2} \sqrt{\beta^{2}-4 \alpha \gamma}\left(x-\lambda t+\xi_{0}\right)\right)\right.
\end{aligned}
$$$$
-\beta)
$$$$
+\frac{\sqrt{30} \sqrt{h c}}{2 c}\left(\sqrt{\beta^{2}-4 \alpha \gamma} \tanh \left(\frac{1}{2} \sqrt{\beta^{2}-4 \alpha \gamma}\left(x-\lambda t+\xi_{0}\right)\right)\right.
$$$$
-\beta)^{2}
$$

where

$$
\lambda=\frac{1}{30} \frac{\sqrt{-30 c h\left(720 \alpha^{2} \gamma^{2} c h^{2}-360 \alpha \gamma \beta^{2} c h^{2}+45 \beta^{4} c h^{2}-30 a c h-10 b^{2} h+3 c g^{2}\right)}}{c h} .
$$

\section{Summary and Discussion}

In conclusion, the improved generalized tanh-coth method is proposed to obtain more general exact solutions of the sixthorder solitary wave equations. It can be observed that the method used is a powerful and more general tool for finding the exact solutions. By using the method, we have obtained new exact solutions in terms of the hyperbolic functions, the trigonometric functions, the exponential functions, and the rational functions. The results reveal that the improved generalized tanh-coth method has significant effects on the wave behavior and can also be applied to other NLEEs in mathematical physics.

The solutions of generalized Riccati equation (7): there are more than seven cases. We can extend the improved generalized tanh-coth method by considering expanding solutions of generalized Riccati equation in this method.

\section{Conflicts of Interest}

The authors declare that there are no conflicts of interest regarding the publication of this paper.

\section{References}

[1] J. S. Russell, "Report on Waves," in Report of the Fourteenth Meeting of the British Association for the Advancement of Science, John Murray, London, UK, 1844.

[2] Z. Zhang and J. Wu, "Generalized $\left(G^{\prime} / G\right)$-expansion method and exact traveling wave solutions of the perturbed nonlinear 
Schrödinger's equation with Kerr law nonlinearity in optical fiber materials," Optical and Quantum Electronics, vol. 49, no. 2, 2017.

[3] A.-M. Wazwaz, "The extended tanh method for new solitons solutions for many forms of the fifth-order KdV equations," Applied Mathematics and Computation, vol. 184, no. 2, pp. 1002 1014, 2007.

[4] O. Guner and A. Bekir, "Traveling wave solutions for timedependent coefficient nonlinear evolution equations," Waves in Random and Complex Media. Propagation, Scattering and Imaging, vol. 25, no. 3, pp. 342-349, 2015.

[5] M. S. Osman, "Nonlinear interaction of solitary waves described by multi-rational wave solutions of the $(2+1)$-dimensional Kadomtsev-Petviashvili equation with variable coefficients," Nonlinear Dynamics. An International Journal of Nonlinear Dynamics and Chaos in Engineering Systems, vol. 87, no. 2, pp. 1209-1216, 2017.

[6] M. S. Islam, K. Khan, and M. A. Akbar, "Application of the improved $F$-expansion method with Riccati equation to find the exact solution of the nonlinear evolution equations," Journal of the Egyptian Mathematical Society, vol. 25, no. 1, pp. 13-18, 2017.

[7] A. Sonmezoglu, M. Ekici, M. Moradi, M. Mirzazadeh, and Q. Zhou, "Exact solitary wave solutions to the new $(3+1)$ dimensional generalized Kadomtsev-Petviashvili equation," Optik-International Journal for Light and Electron Optics, vol. 128, pp. 77-82, 2017.

[8] B. H. Malwe, G. Betchewe, S. Y. Doka, and T. C. Kofane, “Travelling wave solutions and soliton solutions for the nonlinear transmission line using the generalized Riccati equation mapping method," Nonlinear Dynamics. An International Journal of Nonlinear Dynamics and Chaos in Engineering Systems, vol. 84, no. 1, pp. 171-177, 2016.

[9] M. M. Kabir, "Modified Kudryashov method for generalized forms of the nonlinear heat conduction equation," International Journal of the Physical Sciences, vol. 6, no. 25, pp. 6061-6064, 2011.

[10] A. Akbulut, M. Kaplan, and F. Tascan, "The investigation of exact solutions of nonlinear partial differential equations by using $\exp (-\phi(\xi))$ method," Optik-International Journal for Light and Electron Optics, vol. 132, pp. 382-387, 2017.

[11] S. Sahoo and S. Saha Ray, "Lie symmetry analysis and exact solutions of $(3+1)$ dimensional Yu-Toda-Sasa-Fukuyama equation in mathematical physics," Computers \& Mathematics with Applications. An International Journal, vol. 73, no. 2, pp. 253260, 2017.

[12] M. Eslami and H. Rezazadeh, "The first integral method for WuZhang system with conformable time-fractional derivative," Calcolo. A Quarterly on Numerical Analysis and Theory of Computation, vol. 53, no. 3, pp. 475-485, 2016.

[13] W. Cheng and B. Li, "CRE solvability, exact soliton-cnoidal wave interaction solutions, and nonlocal symmetry for the modified Boussinesq equation," Advances in Mathematical Physics, vol. 2016, Article ID 4874392, 7 pages, 2016.

[14] W. Malfliet, "Solitary wave solutions of nonlinear wave equations," American Journal of Physics, vol. 60, no. 7, pp. 650-654, 1992.

[15] E. Fan and Y. C. Hona, "Generalized tanh method extended to special types of nonlinear equations," Zeitschrift für Naturforschung A, vol. 57, no. 8, 2002.

[16] C. A. Gómez and A. H. Salas, "The Cole-Hopf transformation and improved tanh-coth method applied to new integrable system (KdV6)," Applied Mathematics and Computation, vol. 204, no. 2, pp. 957-962, 2008.

[17] A. H. Salas, C. A. Gómez, and J. Castillo, "New solutions for the modified generalized Degasperis-Procesi equation," Applied Mathematics and Computation, vol. 215, no. 7, pp. 2608-2615, 2009.

[18] C. A. Gómez, "Closed form solutions for a generalized Benjamin-BONa-Mahony-BURgers equation with higherorder nonlinearity," Applied Mathematics and Computation, vol. 234, pp. 618-622, 2014.

[19] G. Hernán Garzón and A. G. S. Cesar, "The generalized tanhcoth method applied to biological model referent to nanosolitons of ionic wave," Applied Mathematical Sciences, vol. 9, no. 137-140, pp. 6877-6882, 2015.

[20] M. Torvattanabun and S. Koonprasert, "Variational iteration method combined with the improved generalized tanh-coth method for the generalized $(1+1)$-dimensional and $(2+1)$ dimensional ito equations," Far East Journal of Mathematical Sciences, vol. 100, no. 8, pp. 1339-1355, 2016.

[21] M. A. Christou, "Sixth order solitary wave equations," Wave Motion. An International Journal Reporting Research on Wave Phenomena, vol. 71, pp. 18-24, 2017. 


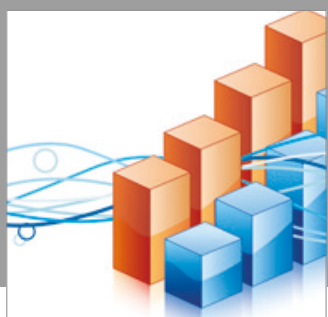

Advances in

Operations Research

vatersals

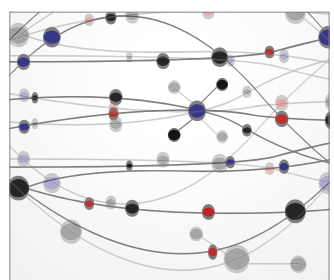

\section{The Scientific} World Journal
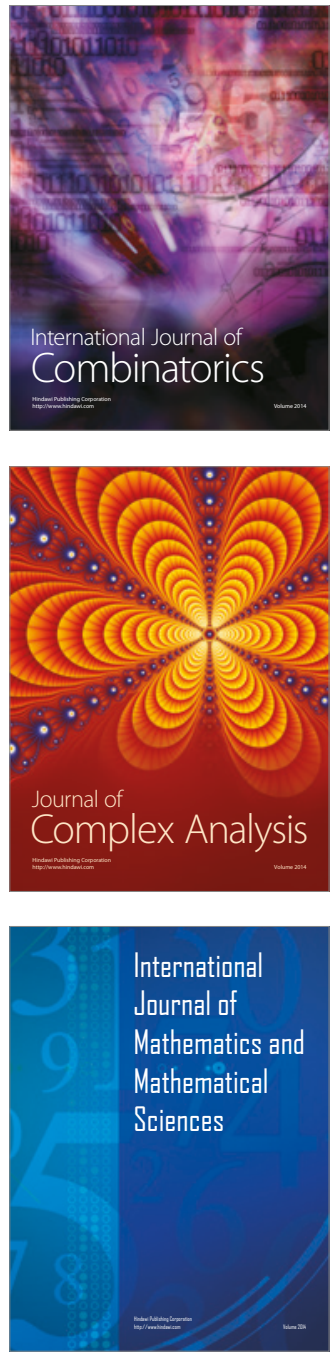
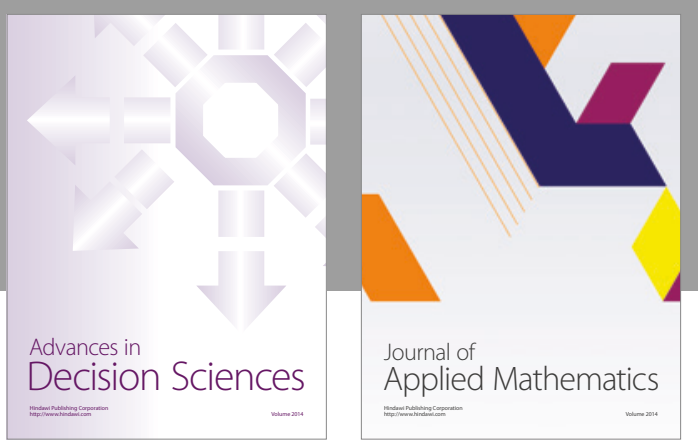

Algebra

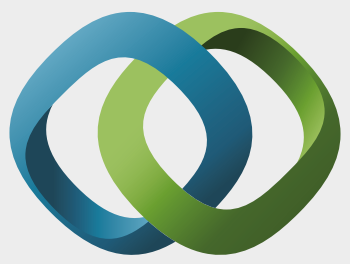

\section{Hindawi}

Submit your manuscripts at

https://www.hindawi.com
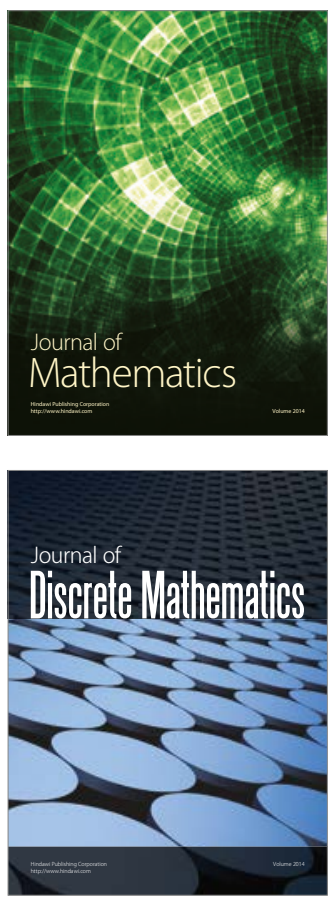

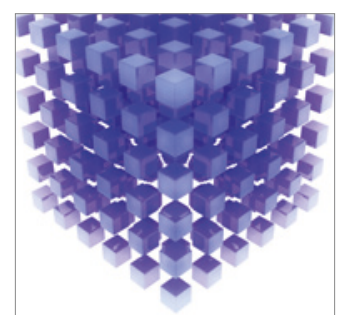

Mathematical Problems in Engineering
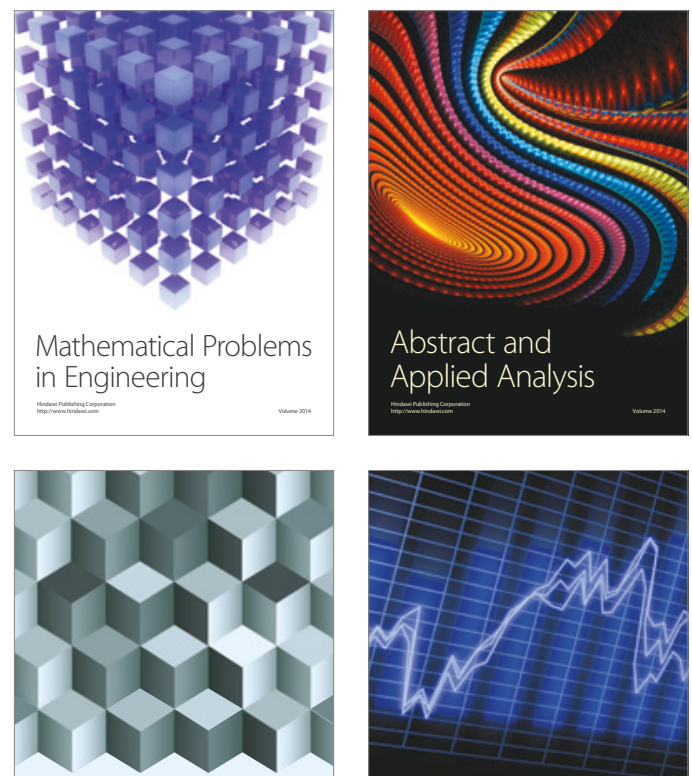

Journal of

Function Spaces

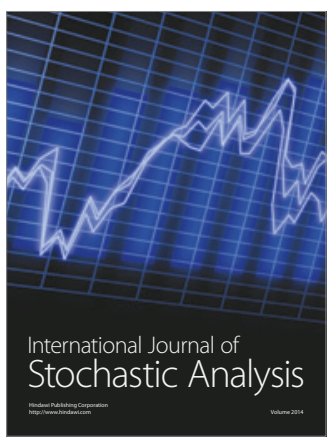

Probability and Statistics
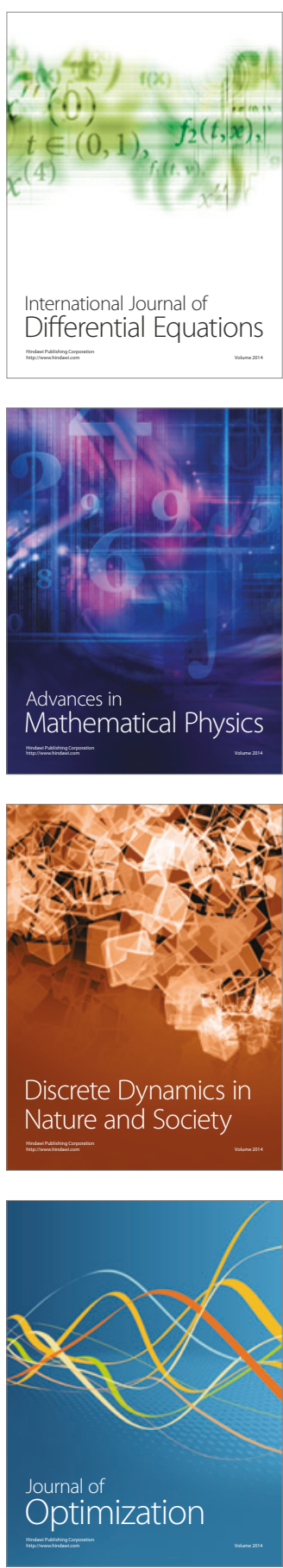